\title{
Correction to: Routine diagnostics for neural antibodies, clinical correlates, treatment and functional outcome
}

\author{
Christian G. Bien ${ }^{1}$ (1) $\cdot$ Corinna I. Bien ${ }^{2} \cdot$ Müjgan Dogan Onugoren ${ }^{3} \cdot$ Desiree De Simoni $^{4,5}$ (D) Verena Eigler ${ }^{6}$. \\ Carl-Albrecht Haensch ${ }^{7}$ (1) Martin Holtkamp ${ }^{8}$. Fatme S. Ismail ${ }^{9}$ (1) Martin Kurthen $^{10} \cdot$ Nico Melzer $^{11}$. \\ Kristina Mayer $^{12}$. Felix von Podewils ${ }^{13}$ (1) . Helmut Rauschka ${ }^{14}$. Andrea O. Rossetti ${ }^{15}$ - Wolf-Rüdiger Schäbitz ${ }^{16}$.

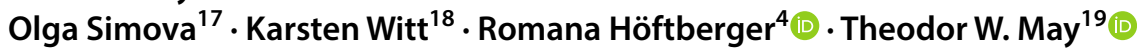

Published online: 12 May 2020

(c) Springer-Verlag GmbH Germany, part of Springer Nature 2020

\section{Correction to: Journal of Neurology} https://doi.org/10.1007/s00415-020-09814-3

The original version of this article unfortunately contained a mistake. The order of the lines in A1 of the Fig. 2 is incorrect.

The corrected Fig. 2 is placed in the following page.

The original article can be found online at https://doi.org/10.1007/ s00415-020-09814-3.

Christian G. Bien

christian.bien@gmx.de

1 Epilepsy Center Bethel, Krankenhaus Mara, Maraweg 17-21, 33617 Bielefeld, Germany

2 Laboratory Krone, Bad Salzuflen, Germany

3 Department of Neurology, Epilepsy Center, Friedrich-Alexan der-Universität, Erlangen-Nürnberg, Germany

4 Division of Neuropathology and Neurochemistry, Department of Neurology, Medical University of Vienna, Vienna, Austria

5 Department of Neurology, University Hospital St. Poelten, St. Poelten, Austria

6 Department of Neurology, Städtisches Klinikum Ludwigshafen Am Rhein, Ludwigshafen, Germany

7 Department of Neurology, Kliniken Maria Hilf Moenchengladbach, Faculty of Health, University of Witten/Herdecke, Moenchengladbach, Germany

8 Epilepsy-Center Berlin-Brandenburg, Institute for Diagnostics of Epilepsy, Evangelisches Krankenhaus Königin Elisabeth Herzberge, Berlin, Germany

9 Department of Neurology, University Hospital Bochum, Knappschaftskrankenhaus, Bochum, Germany
10 Swiss Epilepsy Center, Zurich, Switzerland

11 Department of Neurology with Institute of Translational Neurology, University Hospital Münster, Münster, Germany

12 Department of Neurology, University Hospital of Augsburg, Augsburg, Germany

13 Department of Neurology, University Medicine Greifswald, Greifswald, Germany

14 Department of Neurology and Karl Landsteiner Institute for Neuroimmunological and Neurodegenerative Disorders, Sozialmedizinisches Zentrum Ost, Donauspital, Vienna, Austria

15 Department of Clinical Neurosciences, University Hospital (CHUV) and University of Lausanne, Lausanne, Switzerland

16 Department of Neurology, EvKB-Bethel, Bielefeld, Germany

17 Protestant Hospital Alsterdorf, Epilepsy Center Hamburg, Hamburg, Germany

18 Department of Neurology and Research Centre of Neurosensory Sciences, Carl Von Ossietzky University, Oldenburg, Germany

19 Society of Epilepsy Research, Bielefeld, Germany 
Fig. 2 Disease durations, CSF/ serum/CSF-serum pairs, IgG subclasses, and clinical ratings. a Latency (in months) between disease manifestation and antibody diagnostics in the antibody-positive cases with known disease onset. The lines indicate medians with quartiles. Antibody groups are given in ascending order of their median latencies. $A_{1}$ : linear $x$-axis, $A_{2}$ : logarithmic $x$-axis (note that " 1 " was added to all values to be able to include values of zero). b Ratio of cases with serumonly or CSF-only antibody positivity in the four major antibody groups plus onconeural and GABABR antibodies. The small groups with serumonly and CSF-only findings in the GAD65 group are cases with either very low CSF titers $(N=3)$ and negative serum or serum titers of just 1:500 and negative CSF samples $(N=2)$. In the onconeural group, there was one $\mathrm{Ma} 2$ case that was not fully appreciated in serum (blot positivity only) but clearly diagnosed in CSF (blot and tissue-based assay positive). c IgG subclasses in the four major antibody groups. d Clinical retrospective ratings ("Autoimmune disease of the CNS or PNS?") in descending order of the positive ratings

\section{$A_{1}$}

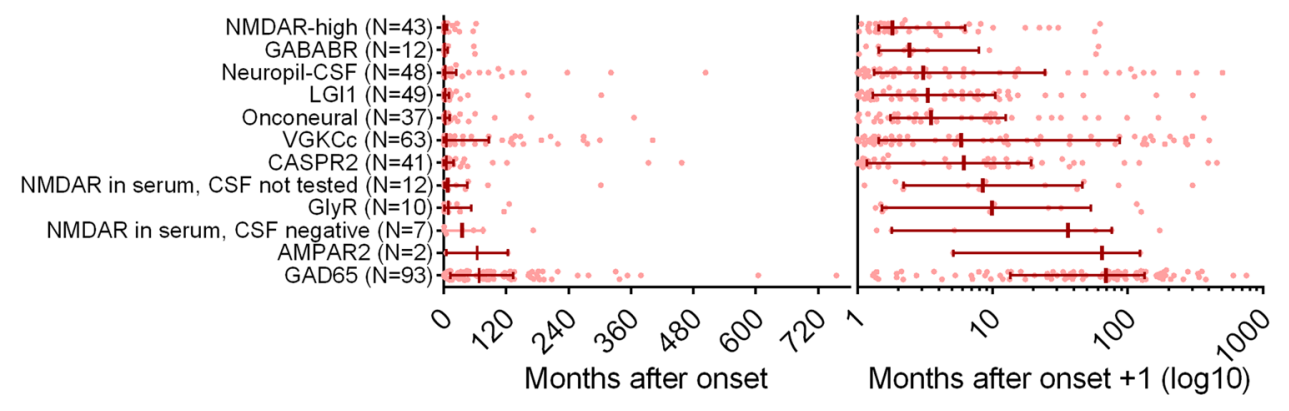

B
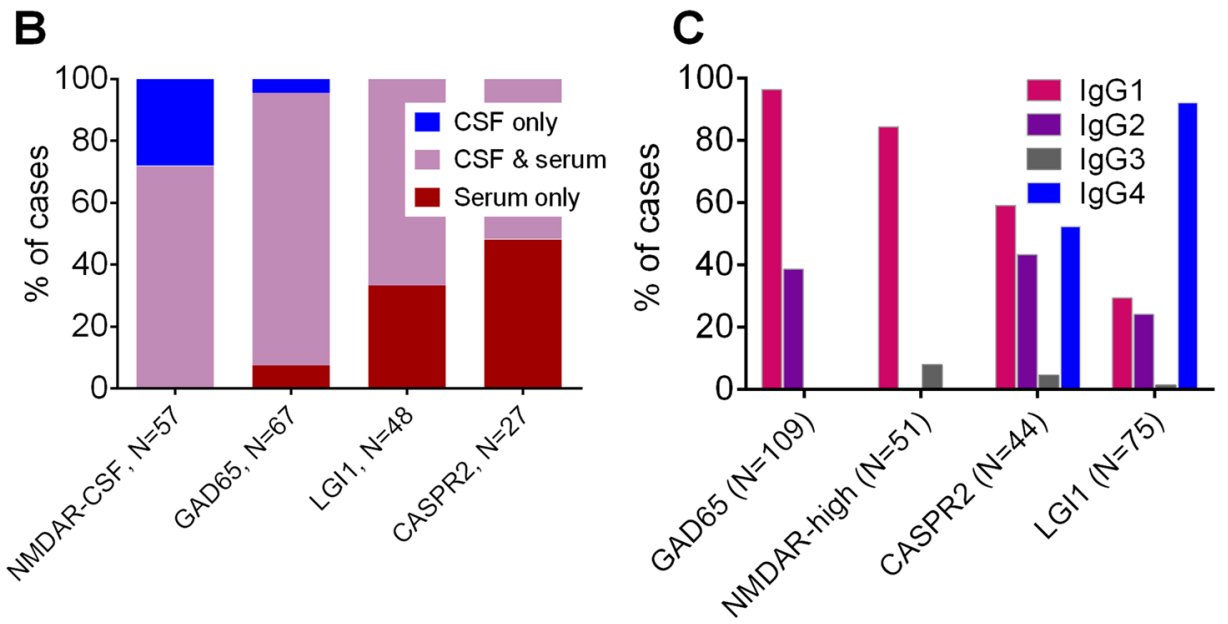

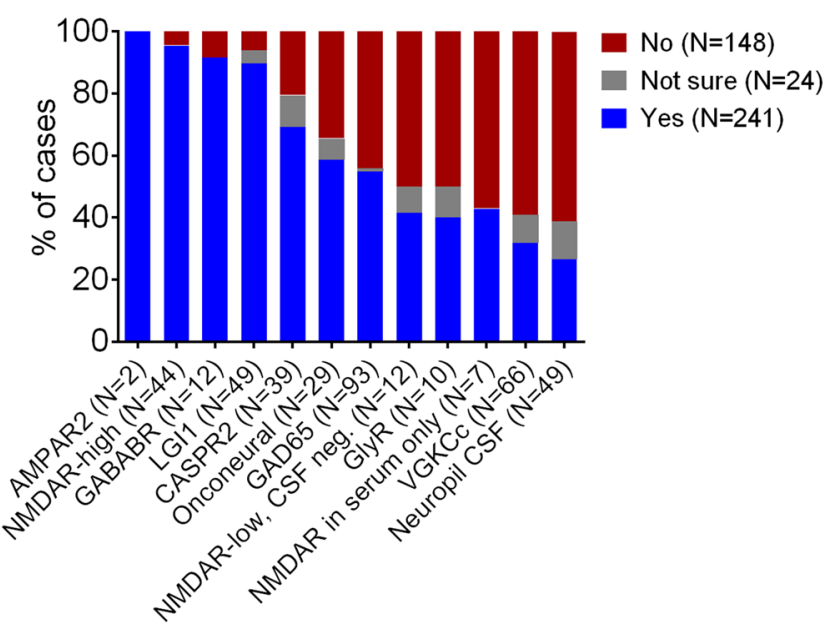

